\title{
Hashimoto Encephalopathy: Impact of Concussion
}

\author{
David S. Younger ${ }^{1,2}$ \\ ${ }^{1}$ Department of Neurology, New York University Langone Health, School of Medicine, New York University, New York, USA \\ ${ }^{2}$ Division of Health, Policy and Management Doctoral Program, School of Public Health, City University of New York, \\ New York, USA \\ Email: david.younger@nyumc.org
}

How to cite this paper: Younger, D.S. (2018) Hashimoto Encephalopathy: Impact of Concussion. World Journal of Neuroscience, 8, 108-112.

https://doi.org/10.4236/wjns.2018.81010

Received: December 29, 2017

Accepted: February 24, 2018

Published: February 27, 2018

Copyright (C) 2018 by author and Scientific Research Publishing Inc. This work is licensed under the Creative Commons Attribution International License (CC BY 4.0).

http://creativecommons.org/licenses/by/4.0/

\begin{abstract}
Patients with Hashimoto encephalitis may present with seizures, stroke-like episodes, transient focal and global neurological deficits, and a variety of neuropsychiatric disturbances. The encephalopathy evolves with elevated anti-thyroid peroxidase antibodies, independent of hormonal thyroid function. A teenager experienced repeated concussions antedating onset of $\mathrm{Ha}$ shimoto encephalopathy. Neuroradiological studies showed overlapping areas of altered brain metabolism and vascular perfusion in the hippocampus, deemed most vulnerable in autoimmune encephalopathy. Concussive brain injury may be a contributing factor in the development of encephalopathy due to its capacity to alter the blood-brain barrier.
\end{abstract}

\section{Keywords}

Autoimmune, Encephalitis, Hashimoto, Encephalopathy, Vasculitis

\section{Introduction}

Hashimoto thyroiditis and encephalopathy are extensively reviewed by Younger [1]. Neurologists have been pursuing the associated but rare encephalopathy associated with Hashimoto thyroiditis for over fifty years. In 1966, the British neurologist, Lord Brain and colleagues [2] described Hashimoto encephalopathy in a 40-year-old man with ictal and stroke-like episodes of confusion and agitation one year after onset of treated hypothyroidism, eventually resolving with the likeliest explanation of an antibody-mediated autoimmune etiopathogenesis. Jellinek and Ball [3] extended the patient of Brain and colleagues [2], who later died at age 62, 12 years after an unrelated cause. Autopsy showed no remaining thyroid tissue and atheromatous cerebrovascular changes with splenic atrophy. 
The authors [3] postulated an autoimmune disturbance as the cause of thyroiditis, encephalopathy, and splenic atrophy. In 2003, Rowland and colleagues [4] described the clinicopathologic findings of all literature cases of Hashimoto encephalopathy beginning with the patient described by Lord Brain and coworkers [2], and adding their own patient.

The diagnosis of Hashimoto encephalopathy rests upon the presence of thyroiditis, with measurably high titers of thyroid peroxidase (TPO) or thyroglobulin ( $\mathrm{Tg}$ ) antibodies, clinical encephalopathy (as evidenced by clouding of consciousness with reduced wakefulness, attention, or cognitive function), and absent evidence of cerebrospinal fluid (CSF) bacterial or viral infection [4]. Historically, as now, it is unknown whether anti-thyroid antibodies and thyroid dysfunction contribute to the pathogenesis of encephalopathy.

\section{Case Report}

A late age teen who met clinical criteria for Hashimoto encephalopathy as described by Rowland and colleagues [4] suffered repeated concussions prior to onset of cognitive and neurobehavioral impairments.

Non-contrast magnetic resonance imaging (MRI) fused to ${ }^{18}$ fluorodeoxyglucose (FDG) positron emission tomography (PET) showed signal abnormality in the left hippocampus tail with hypometabolism in bilateral temporal and parietal lobes (Figure 1). In addition, nuclear medicine cerebral perfusion single photon emission computed tomography (SPECT) of the brain showed overlapping hypoperfusion in bilateral temporal and right parietal lobes.

Serum and cerebrospinal fluid provided to Mayo Laboratories (Rochester, Minnesota) and Athena Diagnostics (Worcester, MA), and analyzed by indirect immunofluorescence assay (IFA) and Western blot for circulating antibodies associated with autoimmune encephalopathy returned negative.

Treatment beginning with weekly intravenous immune globulin (Ig) at the dose of $400 \mathrm{mg}$ per kilogram, was later combined with a tapering course of alternate day prednisone, starting with $60 \mathrm{mg}$, leading to significant improvement.

\section{Discussion}

A relation of Hashimoto encephalopathy to vasculitis was postulated in two cases studied histologically at autopsy showing lymphocytic infiltration of brainstem veins [5], while brain biopsy tissue of another patient showed lymphocytic infiltration of the walls of cerebral arterioles and veins [6]. Brain biopsy tissue in the patient described by Rowland and coworkers [4] showed perivascular cuffs of lymphocytic cells [4].

Ochi and colleagues [7] associated Hashimoto encephalopathy-related pathology and corticosteroid sensitivity with the novel antigen, $\alpha$-enolase, encoded at the 1p36.23 locus. Kishitani and coworkers [8] reported anti-NH2-terminal of $\alpha$-enolase antibodies in $24 \%$ of Hashimoto encephalopathy patients' sera, and identified unilateral and bilateral mesial temporal lobe signal abnormalities on 


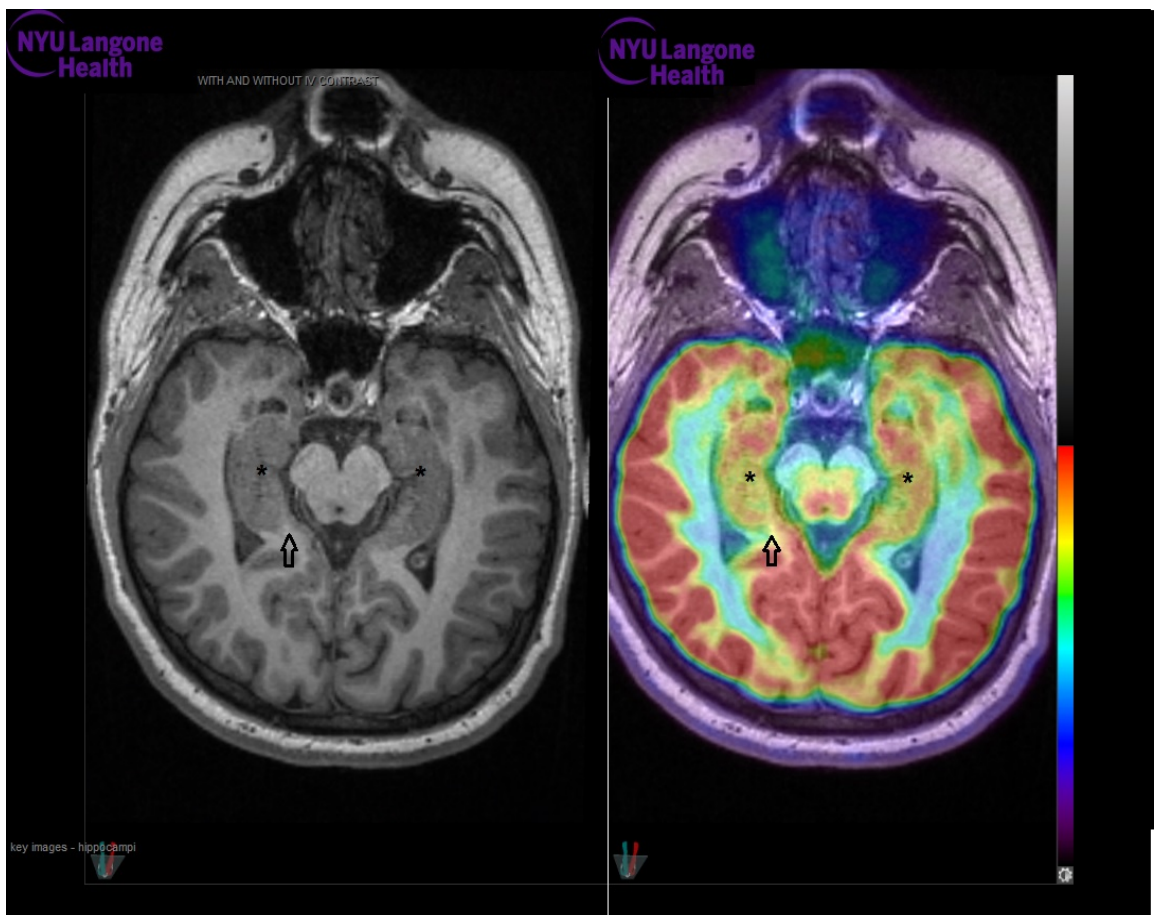

Figure 1. Hashimoto encephalopathy. Positron emission tomographic imaging (right panel) from the vertex to foramen magnum following injection of $10-\mathrm{mCi}{ }^{18}$ fluoro-D-glucose is fused with gadolinium-enhanced magnetic resonance imaging (left panel). There is hypometabolism of the medial temporal lobes (asterisks), and signal change in the tail of the hippocampus (arrows).

brain MRI that were associated with epileptic foci, suggesting a relation to limbic (autoimmune) encephalitis. Although antibodies to $\alpha$-enolase were not determined in the present case, signal abnormality in the tail of the hippocampus, and hypometabolism of the mesial temporal lobes in FDG PET-MRI supports a similar autoimmune mechanism.

Graus and colleagues [9] proposed categorization of cases of Hashimoto encephalopathy as autoimmune encephalitis after exclusion of other syndromes associated with well-defined autoantibodies. The relatively few biopsy and postmortem examinations available in patients with pathogenic serum antibodies have shown infiltrating inflammatory $\mathrm{T}$-cells with cytotoxic granules in close apposition to hippocampal neurons, analogous to microscopic vasculitis [10].

The occurrence of repeated concussions in the present case is intriguing. The mechanisms underlying concussion, mild traumatic brain injury (mTBI) and chronic traumatic encephalopathy are all closely associated with blood-brain barrier (BBB) disruption, as well as, metabolic and morphological brain alterations with activation of triggering receptor expressed on myeloid cells (TREM2), monocyte infiltration and phosphorylated axonal tauopathy [11]. Overlapping abnormalities on SPECT and PET imaging in the present case suggests that BBB disruption might have placed the patient at greater risk for developing autoimmune encephalopathy by allowing passage of circulatory cytokines, autoantibo- 
dies, and other mitigating substances across a disrupted BBB.

Closed-head impact injury in experimental animals is associated with impaired axonal conduction in bilateral mesial hippocampal cells of the stratum alveus [11]. An assay that detects elevated serum levels of ubiquitin C-terminal hydrolase L1 (UCH-L1) and glial fibrillary acidic protein (GFAP) (ClinicalTrials.gov identifier number, NCT01426919) within 12 hours of concussive head injury is associated with neuronal injury and $\mathrm{BBB}$ changes, and predicts the need for neuroradiologic intervention.

\section{Conclusion}

The terms Hashimoto thyroiditis and encephalopathy are deeply entrenched in the scientific literature. While the former is a cornerstone for understanding autoimmune thyroid disease, the latter remains a challenge to clinicians in elucidating the mediating factors that lead to its expression.

\section{Acknowledgements}

The author is grateful to NYU Langone Health in providing ongoing opportunities to investigate the multidisciplinary aspects of autoimmune encephalopathy and concussion. The author expresses appreciation to Eliza B. Geer MD, Memorial Sloan Kettering Cancer Center, Neuroendocrinology, for her assistance in the care of this patient.

\section{Conflict of Interest}

The author denies any conflict of interest.

\section{References}

[1] Younger, D.S. (2017) Hashimoto's Thyroiditis and Encephalopathy. World Journal of Neuroscience, 7, 307-326. https://doi.org/10.4236/wins.2017.73026

[2] Brain, L., Jellinek, E.H. and Ball, K. (1966) Hashimoto's Disease and Encephalopathy. Lancet, 288, 512-514. https://doi.org/10.1016/S0140-6736(66)92876-5

[3] Jellinek, E.H. and Ball, K. (1976) Hashimoto's Disease, Encephalopathy, and Splenic Atrophy. Lancet, 307, 1248. https://doi.org/10.1016/S0140-6736(76)92207-8

[4] Chong, J.Y., Rowland, L.P. and Utiger, R.D. (2003) Hashimoto Encephalopathy. Syndrome or Myth? Archives of Neurology, 60, 164-171. https://doi.org/10.1001/archneur.60.2.164

[5] Nolte, K.W., Unbehaun, A., Sieker, H., et al. (2000) Hashimoto Encephalopathy: A Brainstem Encephalitis? Neurology, 54, 769-770. https://doi.org/10.1212/WNL.54.3.769

[6] Shibata, N., Yamamoto, Y., Sunami, N., et al. (1992) Isolated Angiitis of the CNS Associated with Hashimoto's Disease. Rinsho Shinkeigaku, 32, 191-198.

[7] Ochi, H., Horiuchi, I., Araki, N., et al. (2002) Proteomic Analysis of Human Brain Identifies $\alpha$-Enolase as a Novel Autoantigen in Hashimoto's Encephalopathy. FEBS Letters, 528, 197-202. https://doi.org/10.1016/S0014-5793(02)03307-0

[8] Kishitani, T., Matsunaga, A., Masamichi, I., et al. (2017) Limbic Encephalitis Associated with Anti-NH2-Terminal of $\alpha$-Enolase Antibodies. A Clinical Subtype of 
Hashimoto Encephalopathy. Medicine, 96, e6181.

[9] Graus, F., Titulaer, M.J., Balu, R., et al. (2016) A Clinical Approach to Diagnosis of Autoimmune Encephalitis. Lancet Neurology, 15, 391-404.

https://doi.org/10.1016/S1474-4422(15)00401-9

[10] Bien, C.G., Vincent, A., Barnett, M.H., et al. (2012) Immunopathology of Autoantibody-Associated Encephalitides: Clues for Pathogenesis. Brain, 135, 1622-1638. https://doi.org/10.1093/brain/aws082

[11] Tagge, C.A., Fisher, A.M., Minaeva, O.V., et al. (2018) Concussion, Microvascular Injury, and Early Tauopathy in Young Athletes after Impact Head Injury and an Impact Concussion Mouse Model. Brain, 141, 422-458.

https://doi.org/10.1093/brain/awx350 